\title{
NOTAS SOBRE LA AVIFAUNA DEL HUMEDAL SANTA MARÍA DEL LAGO, CORDILLERA ORIENTAL DE LOS ANDES COLOMBIANOS
}

\author{
Ricardo Álvarez-León \\ Fundación Maguaré. Manizales (Caldas) Colombia \\ alvarez_leon@hotmail.com
}

Manizales, 2008-10-06 (Rev. 2009-11-02)

\section{RESUMEN}

Se presentan los resultados del inventario de la macrofauna observada (desde febrero 23 hasta mayo 31 del 2001) en Santa María del Lago, humedal netamente urbano, de los trece presentes en la ciudad de Bogotá durante su rehabilitación paisajística (recuperación del espejo de agua, revegetalización y reforestación). Los resultados incluyen una lista con las observaciones sobre el hábitat, actividad y frecuencia de tres especies de peces, una de anfibios, treinta y una de aves y tres de mamíferos, registradas en los diferentes muestreos mensuales con una intensidad de uno por semana. Dentro de la fauna del citado humedal, se resalta la presencia de los mamíferos Cavia porcellus (Caviidae), Oeomys speciosus (Muridae) y Olygoryzomys fulvescens (Muridae), la rana Hyla labialis (Hylidae) y los peces Eremophilus mutissii (Trichomycteridae) y Grundulus bogotensis (Characidae), que se consideraban desaparecidos del humedal. También se registran por primera vez para el humedal, nueve especies de aves (Anas platyrhynchos, Carduelis psaltria, Anser anser, Diglossa humeralis, Piranga olivacea, P. rubra, Rhamphocelus dimidiatus, Stelgidopteryx ruficollis, Vireo olivaceus), al igual que el pez exótico (C. auratus).

\section{PALABRAS CLAVE}

Aves acuáticas, Aves continentales, Vertebrados, Humedales andinos, Colombia.

\section{ABSTRACT}

The results of the inventory of the observed macrofauna are presented (February 23 - May 31 of the 2001) in Santa María del Lago, one of the thirteen present wetlands in Bogotá during their rehabilitation landscape (recovery of the mirror of water, revegetation and reforestation). The results include a list with the observations about the habitat, activity and frequency of 3 species of fish, 1 of amphibians, 31 of birds and 3 of mammals, registered in the different monthly samplings with an intensity of one per week. Inside the fauna of the mentioned wetland, the presence of the mammals Cavia anolaimae (Caviidae), Oeomys speciosus (Muridae) and, Olygoryzomys fulvescens (Muridae), the frog Hyla labialis (Hylidae) and the fishes Eremophilus mutissii (Trichomycteridae) and, Grundulus bogotensis (Characidae) that were considered missing of the wetland. Also report for first time of the wetland, 9 species of birds (Anas platyrhynchos, Carduelis psaltria, Anser anser, Diglossa humeralis, Piranga olivacea, P. rubra, Rhamphocelus dimidiatus, Stelgidopteryx ruficollis, Vireo olivaceus), and exotic fish (C. auratus).

\section{KEY WORDS}

Waterbirds, Continental birds, Vertebrates, Andean wetlands, Colombia.

Tal como ocurre con otras áreas, el inventario de la avifauna de un área urbana es complejo. Inciden en este problema la ausencia actual de investigaciones que permitan realizar un seguimiento adecuado a lo largo del año a través de inventarios y colecciones sistemáticamente organizadas. Sin embargo, existen del pasado algunas investigaciones ornitológicas que han registrado aves residentes y migratorias para el Humedal Santa María del Lago (Rodríguez-Mahecha et al., 2000; Calvachi-Zambrano, 2003). Otras investigaciones como los Conteos de Aves de los Humedales de Bogotá y los Conteos Navideños de Aves, realizados por la Asociación Bogotana de Ornitología ABO, sólo están disponibles entre 1998 y 2000. Recientemente, Cely et al. (2002), en Santa María del Lago (2 de febrero), durante el II Conteo de Aves en los Humedales de la Sabana de Bogotá, organizado por la ABO y el I Censo Nacional de Aves organizado por el Instituto de investigaciones Biológicas "Alexander von Humboldt" y Calidris, registraron seis especies de aves acuáticas, entre ellas una migratoria; aunque el número de especies observadas prácticamente se duplicó, hubo una leve 
disminución del número total de individuos. Por ejemplo, la población de fochas (Fulica americana) bajó, pues pasó de sesenta y ocho a cero individuos, en la de la tingua azul (Porphyrula martinica) de quince a cero individuos y en la del chamón (Molothrus bonariensis) que pasó de cincuenta y cinco a tres individuos. No obstante, la población del zambullidor piquipinto (Podilymbus podiceps) aumentó de dos a diez y siete individuos y se registró por primera vez al pato canadiense o cerceta aliazul (Anas discors).

Revisando la literatura existente se ve que entre 1930 y 1950 el humedal realizaba sus funciones en armonía con el ambiente circundante; entre 1950 y 1960 la construcción de la carrera 76 dividió el humedal (Parque del Lago y Parque Tabora); entre 1960 y 1980 la eutroficación del humedal ya era evidente debido a la construcción de la calle 80, la construcción de la Escuela Distrital del Niño, el parqueadero de buses troleys y la urbanización acelerada de los alrededores; entre 1980 y 1990 rellenos acelerados con escombros y basuras, dragados totales en 1986, construcción del Conjunto Residencial SAGO, adecuación sanitaria, vía perimetral automotor, desaparición de flora y fauna; entre 1990 y 2000 el deterioro generalizado por la acumulación de escombros y basuras, quemas, indigencia, libre acceso, lavado de caballos, campo de entrenamiento de perros de vigilancia privada, tala de árboles de la escuela distrital vecina (calle 80-carrera 76) en 1992, la recuperación sanitaria de la CAR en 1993, cerca de ladrillo $(1 \mathrm{~m})$ y malla aldabonada $(2 \mathrm{~m})$ en 1995 , la construcción del Conjunto Residencial San Francisco en 1996, el espejo de agua alcanza su máxima eutroficación.

El presente estudio hizo parte integral del Proyecto "Recuperación del Humedal de Santa María del Lago, EAAB / DAMA" y registra la macrofauna observada en las áreas de enea (Typha domingensis), buchón (Eichhornia crassipes), pasto kikuyo (Pennisetum clandestinum); árboles nativos como el sauce (Salix humboltiana), el cerezo (Prunus serotina), el aliso (Alnus acuminata), el arrayán (Myrcianthes leucoxyla); árboles exóticos introducidos como los urapanes (Fraxinus chinensis), las acacias (Acacia spp.), los eucaliptus (Eucaliptus sp.), las higuerillas (Ricinus comunis) y los chilcos (Baccaris latifolia); así como en los pantanos arbolados, matorral de pantano, pantano herbáceo, praderas emergentes graminoide, juncoide, praderas y caños, donde es característica la vegetación asociada a las aguas dulces. La estratificación habitacional de las especies observadas se adapta a los hábitats acuáticos y de pantanos herbáceos de la Sabana de Bogotá, específicamente del Humedal de Santa María del Lago, las cuales incluyen según Schmidt-Mumm (1998):

Praderas Emergentes de Forbias Bajas: frecuentes, donde las formas de crecimiento dominantes presentan una altura menor de $1.5 \mathrm{~m}$ cuando se encuentran en pleno desarrollo, por lo general estas comunidades se encuentran caracterizadas en la Sabana de Bogotá como Bidens laevis, Polygonum hydropiperoides, P. punctatum, Rumex conglomeratus.

Praderas Emergentes Graminoide Intermedia: ocasionales, donde la fisionomía se caracteriza por la vegetación de apariencia graminoide que presenta normalmente una altura entre 0.50 y 2 $\mathrm{m}$. Como representantes típicos de esta formación se pueden mencionar las comunidades de Typha angustifolia o T. cf. latifolia.

Praderas Emergentes Graminoide Baja: raras, donde la fisionomía se caracteriza por una vegetación de apariencia graminoide que presenta normalmente una altura menor de los 0.50 $\mathrm{m}$. Entre las más representativas, y como manchas locales, se pueden encontrar algunas comunidades en los cuerpos de la Sabana de Bogotá de Cyperus rufus, Glyceria septentrionalis o Leersia hexandra.

Praderas Emergentes Juncoide Alta: ocasionales, donde la fisionomía se caracteriza por la vegetación de apariencia juncoide que alcanza normalmente una altura superior a los $1.5 \mathrm{~m}$. La comunidad de esta formación, caracterizada por el junco Scirpus californicus, se distingue en la mayoría de los cuerpos de agua de la Sabana de Bogotá y en algunos humedales la fisionomía domina gran parte del paisaje.

Praderas Emergentes Juncoide Intermedia: ocasionales, donde la fisionomía se caracteriza por la vegetación de apariencia juncoide que presenta normalmente una altura entre 0.30 y $1.5 \mathrm{~m}$. La fisionomía de estas comunidades se caracteriza por parches locales de Juncus densiflorus, J. effuscus, J. microcephalus.

Praderas Flotantes de Forbias Bajas: comunes, donde las formas de crecimiento dominantes presentan una altura menor de $1.5 \mathrm{~m}$ cuando se encuentran en pleno desarrollo. Especies postradas sobre el espejo de agua como Ludwigia peploides o Hydrocotyle ranunculoides 
representan un estado pionero de una pradera. En una fase más compacta y consolidada se presenta Bidens laevis.

Praderas Errantes Emergidas de Comunidades Taloides: frecuentes, donde la fisionomía está caracterizada por plantas de estructura reducida o taloide. Esta vegetación, fácilmente dispersable por el viento y usualmente protegida por vegetación enraizada emergente, representa la mayoría de las especies acropleustófitas en la Sabana de Bogotá. Las comunidades más representativas corresponden a manchas homogéneas o a mezcla de especies como Azolla filiculoides, Lemma gibba, L. minuta, Spirodela intermedia o Ricciocarpus natans, entre otras.

Praderas Errantes Emergidas de Comunidades Foliosas: frecuentes, donde la fisionomía conspicua se caracteriza por plantas de apariencia herbácea. Originalmente representada por comunidades de Limnobium laevigatum. Sin embargo, con la introducción de Eichhornia crassipes, se ha logrado observar un dramático aumento de este tipo de pradera errante usualmente facilitada también por el aporte de nutrientes a los cuerpos de agua. Frecuentemente estas praderas son invadidas por Ludwigia peploides o Hydrocotyle ranunculoides y compactadas a manera de pradera flotante, la cual proporciona luego un substrato para una colonización secundaria, inclusive de especies enraizadas emergentes poco frecuentes como la begonia de pantano Begonia fisheri o la orquídea de pantano Habenaria repens.

Praderas Errantes Sumergidas de Comunidades Taloides: ocasionales, donde la fisionomía se caracteriza por plantas de estructura reducida o taloide. Estas comunidades errantes se pueden encontrar en el cuerpo de agua de forma abierta y dispersa o en parches más o menos compactos, tanto expuestos al sol como entre y debajo de la vegetación errante emergida. Entre las más representativas y que pasan muchas veces desapercibidas se pueden mencionar las comunidades de Wolffia columbiana, Wolffiella lingulata, W. oblonga.

Praderas Errantes Sumergidas de Comunidades Foliosas: raras, donde la fisionomía conspicua se caracteriza por plantas de apariencia herbácea. Está bien representada en el piso térmico cálido por especies de Utricularia o Ceratophyllum, en la Sabana de Bogotá escasamente se pueden observar algunas poblaciones de U. gibba.

\section{MATERIAL Y MÉTODOS}

Se utilizó el método de observación directa con binoculares de 7 × 50 para identificar las aves, los anfibios y los mamíferos, así como la extracción con redes de los peces. Para asegurar la identidad de los organismos, se consultaron trabajos y claves sobre peces (Dahl, 1971), aves (Meyer de Schauensee, 1979; Phelps \& Meyer de Schauensee, 1979; Rodríguez-Mahecha, 1982; Hilty \& Brown, 1986; Zerda, 1999), anfibios (Calvachi-Zambrano, 2003) y mamíferos (Rodríguez-Mahecha et al., 1982; Pinto et al., 2001); se anotó el hábitat, la actividad y la abundancia de los individuos.

Las salidas de campo se llevaron a cabo en los seis sectores principales en los cuales se dividió el humedal, con una periodicidad semanal, durante cuatro meses, lo que permitió tener un estimado de la abundancia de las familias y especies de aves, en los distintos hábitats y sectores. Las observaciones se realizaron caminando alrededor del humedal y esporádicamente también desde un bote inflable.

Los seis sectores en los cuales se dividió el humedal fueron visitados y recorridos semanalmente durante cuatro meses, es decir, las evaluaciones coincidieron con el período durante el cual se realizó la recuperación y adecuación del humedal. Los sectores fueron caracterizados de acuerdo a los criterios de Schmidt-Mumm (1998), teniendo en cuenta el arbolado, los matorrales, el pantano herbáceo, las praderas emergentes y los caños presentes. La estratificación habitacional de las especies observadas se encuentra adaptada a los hábitats acuáticos y de pantanos herbáceos del Humedal de Santa María del Lago.

Sector 1. Desprovista de vegetación alta, a excepción de 10 a 12 eucaliptos con altura de 10 a $12 \mathrm{~m}$. que bordean el terreno que ocupa la Escuela Distrital del Niño; las riberas tienen una suave pendiente que oscila entre 20 y $30^{\circ}$. En general, el espejo de agua tiene praderas emergentes de forbia bajas, las cuales se extienden hasta el límite de la Zona 2, donde comienzan a aparecer praderas emergentes graminoide intermedias. 
Sector 2. Con vegetación típica de praderas emergentes graminoide intermedias, aparición de islotes conformados por praderas emergentes graminoide baja en la periferia y de praderas emergentes juncoide intermedia. Abunda el pasto kikuyo en las áreas aledañas a la vegetación que bordea el espejo de aguas del humedal y éste comienza a presentar los efectos del buchón introducido Eichhornia crassipes, al formar una pradera errante incrementada por aporte de nutrientes del cuerpo de agua y la invasión de otras plantas que, compactadas, forman praderas flotantes.

Sector 3. Con vegetación aislada y de talla media en sus riberas, éstas tienen una suave pendiente que oscila entre 30 y $35^{\circ}$, el cuerpo de agua tiene una forma elipsoidal y en ella desemboca uno de los dos caños de aguas grises (lluvias y negras) que surten de agua al humedal, las praderas emergentes graminoide intermedias se ven invadidas de matorrales bajos, típicos de las praderas emergentes juncoide intermedias.

Sector 4. Con vegetación alta y media de varias especies de árboles, la cual domina las riberas y áreas aledañas al espejo de agua del humedal, a la carrera 76 y a la calle 75 . La ribera es escarpada y con pendientes que fluctúan entre 45 y $90^{\circ}$. El espejo de agua tiene praderas emergentes de forbia bajas y la invasión de otras plantas que, compactadas, forman una pradera flotante. Aparecen islotes conformados por praderas emergentes graminoide intermedias.

Sector 5. Con vegetación alta de varias especies de árboles, la cual domina las riberas y áreas aledañas al espejo de agua del humedal, las riberas son de pendiente suave entre 20 y $30^{\circ}$. El espejo de agua tiene praderas emergentes de forbia bajas y la invasión de otras plantas que, compactadas, forman una pradera flotante, así como los efectos del buchón (E. crassipes) al formar una pradera incrementada por aporte de nutrientes del cuerpo de agua. Aparecen islotes de conformados por praderas emergentes juncoide intermedia (Juncus spp.).

Sector 6. Desprovista de vegetación alta a excepción de pinos que bordean los terrenos que ocupa el Conjunto Residencial SAGO y la carrera 75; existen matorrales aislados en la zona limítrofe con el Conjunto Residencial San Francisco. En general, cerca al espejo de agua, lo típico es el pasto kikuyo. El espejo de agua tiene forma elipsoidal y en el extremo nororiental desemboca el segundo caño de aguas grises que alimentan el humedal; las riberas tienen una suave pendiente que oscila entre 20 y $30^{\circ}$. La vegetación típica es de praderas errantes emergidas de comunidades taloides y la de praderas errantes emergidas de comunidades foliosas donde sobresalen y dominan los islotes de buchón ( $E$. crassipes) que son transportados por el viento, ofreciendo diversos paisajes dependiendo de la intensidad y la ribera que ocupen.

Análisis puntuales se realizaron por la firma Hidroanálisis-Prodycon Ltda., durante la tercera semana de febrero del 2001, en tres estaciones: (1) entrada del efluente principal -esquina carrera 76-, (2) punto medio aproximado del humedal- frente a la calle 77-, (3) salida del humedal -único afluente-. Dentro del humedal, y por los estudiantes de la Facultad de Biología Marina de la Universidad Jorge Tadeo Lozano en mayo del 2001, se realizaron análisis en dos estaciones y fueron comparados con los realizados en años pasados por diferentes autores. Finalmente, se analizaron y discutieron los antecedentes de la medición puntual de dichos parámetros fisicoquímicos -sobre la calidad de las aguas del humedal- con el realizado durante el desarrollo de la presente investigación.

\section{RESULTADOS}

En el Apéndice 1 se encuentra el resumen de las especies, el nombre vernacular, su categoría respecto al humedal y las fuentes previas. Así mismo, en el Apéndice 2 se encuentra la comparación de las diferentes evaluaciones de carácter físico, químico y microbiológico.

A continuación se incluye la lista de familias y especies, con el nombre científico y el nombre vernacular, las zonas, la fecha de observación (día, mes, año), la cantidad de individuos y la actividad en la cual se les observó: en percha (p), caminando (c), volando (v), acicalando (a), el estado de desarrollo, enfatizando en la presencia de juveniles (juv.), así como los comentarios de los autores que han trabajado sobre la especie en el Humedal Santa María del Lago.

\section{THROCHILIDAE}

Colibri coruscans (Gould, 1846); nv. Colibrí orejiverde, Orejivioleta vientreazul, Tominejo, Sparkling violetear. 
Se observaron 11 individuos, en los sectores 1 (10-04-01, 1p ) y 4 (23-02-01, 3 p; 30-03-01, 2 p; 06-04-01, 3 p; 10-04-01, 2 p). Previamente Molina \& Osorio (1995) у Benítez-Castañeda (2000a) la habían citado para el humedal.

\section{COEREBIDAE}

Diglossa humeralis (Fraser, 1840); nv. Carbonero común, Pinchaflor negro, Black flowerpiercer.

Se le observó en los sectores 1 (04-10-03, 2 p) y 4 (02-23-01, 3 p; 03-30-01, 2 p; 04-06-01, 3 p; 04-10-03, 2 p). No había sido previamente registrada para el humedal, por lo que se constituye en un nuevo registro.

\section{ARDEIDAE}

Bubulcus ibis (Linnaeus, 1758); nv. Garcita del ganado, Garcilla bueyera, Castle egret.

Se observaron 23 individuos en los sectores 2 (23-03-01, 1 v), 3 (09-03-01, 1 v; 14-03-01, 2 c; 06-04-01, 1 v), 4 (23-02-01, 1 v; 02-03-01, 1 v; 30-03-01, 1 v), 5 (14-03-01, 2 v; 30-03-01, 1 v; 06-04-01, 2 v; 10-04-01, 4 c) y 6 (14-03-01, 1 v; 23-03-01, 3 v; 30-03-01, 1 v; 10-04-01, 1 v). Previamente Valencia (1999) y Benítez-Castañeda (2000a) la habían citado para el humedal.

Butorides striatus (Linnaeus, 1758); nv. Garza castaña, Garcilla cuelligrís, Striated heron.

Se observaron 6 individuos, en los sectores 1 (02-03-01, 1 juv. v, 1 c; 09-03-01, 1c), 2 (30-0301, 1 v), 3 (10-04-01, 1v) y 5 (14-03-01, 1v). Previamente Valencia (1999) y Benítez-Castañeda (2000a) la habían citado para el humedal.

Egretta alba (Linnaeus, 1758); nv. Garza real, Garza blanca, Garceta real, Great egret.

Se observaron 10 individuos en los sectores 1 (02-03-01, 3 c; 09-03-01, 3 c), 3 (09-03-01, 2 c), $5(14-23-01,1$ v) y 6 (30-03-01, 1 c). Previamente Valencia (1999) y Benítez-Castañeda (2000a) la habían citado para el humedal.

ICTERIDAE

Agelaius icterocephalus (Linnaeus, 1766); nv. Monjita bogotana, Tordo capuchidorado, Yellowhooded blackbird.

Se observaron 68 individuos en los sectores 1 (23-02-01, 2 m p, 2 h v, 1 h c; 14-03-01, 2 m v, 1 h p, 1 m p; 06-04-01, 1 m p; ), 3 (23-02-01, 1 m p; 09-03-01, 1 m p; 30-03-01, 1 m p; 30-03-01, $1 \mathrm{~m} \mathrm{p} ;$ 10-04-01, $1 \mathrm{~m} \mathrm{v}$ ), 4 (23-02-01, 4 m p; 09-03-01, 7 m c, 1 m p; 30-03-01, 4 m v, 1 m p), 5 (23-02-01, 4 m v; 02-03-01, 2 m p; 09-03-01, 2 m v, 7 m p; 14-03-01, 2 m v; 23-03-01, 2 m v, 1 h p; 30-03-01, 1 m v, 2 m p, 1 h p; 10-04-01, 1 m p) y 6 (23-02-01, 4 m p, 1 h v; 02-03-01, 1 m p; 09-03-01, 1 m v; 14-03-01, $1 \mathrm{~m} \mathrm{p;} \mathrm{23-03-01,} 1$ m v; 30-03-01, 1 m p; 30-03-01, 1 m p). Previamente Molina \& Osorio (1995), Valencia (1999) y Benítez-Castañeda (2000a) la habían citado para el humedal.

Molothrus bonariensis (Gmelin, 1789); nv. Maicero, Chamón, Carbonero, Vaquero lustroso, Shiny cowbird.

Se observaron 70 individuos en los sectores 1 (23-02-01, 1 v; 02-03-01, 2 v, 3 p), 2 (14-03-01, 3 v), 3 (02-03-01, 1 p; 09-03-01, 4 p; 14-03-01, 6 v; 23-03-01, 2 p; 30-03-01, 3 v; 2 p), 4 (23-0201, 32 c; 09-03-01, 3 p; 23-03-01, 1 v), 5 (09-03-01, 1 v, 2 a; 30-03-01, 1 v; 06-04-01, 1 m p) y 6 (30-03-01, 1 c; 10-04-01, 1 c). Previamente Valencia (1999) y Benítez-Castañeda (2000a) la habían citado para el humedal.

\section{COLUMBIDAE}

Columba livia (Gmelin, 1879); nv. Paloma común, Paloma de castilla, Zuro, Rock dove.

Se observaron 116 individuos, en los sectores 1 (09-03-01, 1 c; 14-03-01, 4 p); 2 (09-03-01, 6 c), 4 (02-23-01, 24 v, 6 p; 09-03-01, 6 c, 1 p; 14-03-01, 24 v., 5 p; 23-03-01, 1 v, 6 p); 5 (09-0301, 3 v, 5 p; 14-03-01, 2 v), y 6 (09-03-01, 6 v; 14-03-01, 2 p; 23-03-01, 1 v). Previamente Benítez-Castañeda (2000) la había citado para el humedal.

Zenaida auriculata (Des Murs, 1847); nv. Torcaza bogotana, Paloma sabanera, Torcaza, Collareja, Eared dove. 
Se observaron 133 individuos en los sectores 1 (06-04-01, 4 v, 8 p; 10-04-01, 1 c, 5 v, 1 p; ); 2 (06-04-01, 2 v; ), 3 (30-03-01, 2 v; 10-04-01, 2 v; ) y 4 (30-03-01, 16 p; 06-04-01, 6 v; 10-04-01, 8 v, 10 p); 5 (30-03-01, 28 v; 06-04-01, 2 c, 3 v; 10-04-01, 6 v, 1 p) y 6 (30-03-01, 14 v; 06-0401, 6 v; 10-04-01, 8 v). Previamente Valencia (1999) y Benítez-Castañeda (2000a) la habían citado para el humedal.

\section{PSITTACIDAE}

Forpus conspicillatus (Lafresnaye, 1848); nv. Periquito de anteojos, Cascabelito, Spectacled parrotlet.

Se observaron 2 individuos en el sector 5 (23-02-01, 2 v). Previamente Valencia (1999) la había citado para el humedal.

HIRUNDINIDAE

Hirudo rustica (Linnaeus, 1758); nv. Golondrina negra, Golondrina tijereta, Barn swallow.

Se observaron 40 individuos en los sectores 1 (09-03-01, 6 v), 2 (23-02-01, 2 v; 09-03-01, 2 v; 06-04-01, 1 v), 3 (23-02-01, 14 v; 30-03-01, 2 v; 06-04-01, 12 v), 4 (23-03-01, 2 v; 30-03-01, 4 v; 06-04-01, 20 v; 10-04-01, 4 v), 5 (02-03-01, 7 v; 30-03-01, 3 v; 06-04-01, 18 v; 10-04-01, 8 v) y 6 (02-03-01, 16 v; 23-03-01, 18 v; 10-04-01, 6 v). Previamente Valencia (1999) y BenítezCastañeda (2000a) la habían citado para el humedal.

Notocheliodon murina (Cassin, 1853); nv. Golondrina vientrigris, Golondrina, Brown-bellied swallow.

Se observaron 40 individuos en los sectores 2 (23-02-01, 2 v; 09-03-01, 2 v), 3 (23-02-01, 14 v) y 5 (23-02-01, 16 v). Previamente Valencia (1999) y Benítez-Castañeda (2000a) la habían citado para el humedal.

Stelgidopteryx ruficollis (Vieillot, 1817); nv. Golondrina pecho marrón.

Se observaron 115 individuos en los sectores 2 (14-03-01, 2 v; 30-03-01, 1 v), 3 (14-03-01, 15 v; 30-03-01, 20 v; 10-04 -01, 5 v), 4 (30-03-01, 7 v; 06-04-01, 2 v; 10-04-01, 4 v), 5 (14-03-01, 20 v; 30-03-01, 2 v; 06-04-01, 20 v; 10-04-01, 8 v) y 6 (14-03-01, 2 v; 23-03-01, 4 v). No había sido previamente citada para el humedal.

\section{TURDIDAE}

Turdus fuscater d'Orbigny \& Lafresnaye, 1837; nv. Mirla, Mirlo grande, Great thrush.

Se observaron 155 individuos en los sectores 1 (02-03-01, 2 v, 1 p; 09-03-01, 1 c; 14-03-01, 1 v, 1 p; 23-03-01, 5 v; 06-04-01, 14 p); 2 (04-10-03, 2 p; 09-03-01, 2 p; 14-03-01, 1 v; 23-03-01, 1 v; 06-04-01, 1 v, 1 p; 10-04-01, 1 p); 3 (09-03-01, 1v; 14-03-01, 2 v; 06-04-01, 1 c, 1v; 10-0401, 2 p); 4 (23-02-01, 4 c; 02-03-01, 2 c; 09-03-01, 1 v; 10-04-01, 1 v); 5 (23-02-01, 7 c, 9 p; 0203-01, 1 p; 09-03-01, 3 v, 11 p; 14-03-01, 5 v; 06-04-01, 1 c, 1 v, 2 p); y 6 (02-03-01, 1 p; 14-0301, 3 c, 1 p; 23-03-01, 1 v; 30-03-01, 1 c; 06-04-01, 1 c; 10-04-01, 2 c, 1 p). Previamente Benítez-Castañeda (2000a) la había citado para el humedal.

\section{RALLIDAE}

Fulica americana (Gmelin, 1758); nv. Tingua pico amarillo, Focha americana, American coot.

Se observaron 383 individuos en los sectores 1, 2, 3, 4, 5, 6. Previamente Molina \& Osorio (1995), Valencia (1999) y Benítez-Castañeda (2000a) la habían citado para el humedal.

Gallinula chloropus (Linnaeus, 1758); nv. Tingua pico rojo, Gallareta piquiroja, Common gallinule.

Se observaron 107 individuos en los sectores 1, 2, 3, 4, 5, 6. Previamente Molina \& Osorio (1995), Valencia (1999) y Benítez-Castañeda (2000a) la habían citado para el humedal.

Porphirio martinicus (Linnaeus, 1756); nv. Tingua pico azul, Gallareta morada, Purple gallinule.

Se observaron 104 individuos en los sectores 1, 2, 3, 4, 5, 6. Previamente Molina \& Osorio (1995), Valencia (1999) y Benítez-Castañeda (2000a) la habían citado para el humedal.

PODICIPEDIDAE 
Podilymbus podiceps (Linnaeus, 1758); nv. Zambullidor pico rayado, Patico zambullidor, Zambullidor piquipinto, Pied-billed grebe.

Se observaron 17 individuos en los sectores 1 (23-02-01, 2 n; 02-03-01, 1 n; 09-03-01, 1 n), 2 (23-02-01, 2 n, 1 juv. n; 02-03-01, 1 juv. n; 14-03-01, 2 n; 23-03-01, 1 n; 10-04-01, 1 n), 3 (2303-01, 2 n; 10-04-01, 1 n), 4 (30-03-01, 1 n), y 6 (30-03-01, 1 n; 06-04-01 1 n). Previamente Valencia (1999) y Benítez-Castañeda (2000a, 2000b, 2001) la habían citado para el humedal.

ANATIDAE

Anas platyrhynchos (Linnaeus, 1758); nv. Pato Doméstico, Pato cabeciverde.

Se observaron 22 veces en los sectores 1 (30-03-01, 3 a; 06-04-01, 5 a; 10-04-01, 5 a), 3 (0604-01, 2 c; 10-04-01, 4 a) y 4 (10-04-01, 3 n). No había sido previamente registrada para el humedal, por lo que se constituye en un nuevo registro.

Anser anser (Linnaeus, 1758); nv. Ganso común, Ganso europeo.

Se observaron 68 veces en los sectores 1 (23-02-01, 1 n; 02-03-01 4 n; 09-03-01, 2 n; 14-0301, 2 n; 23-03-01, 4 a.; 06-04-01, 7 a; 10-04-01, 7 c), 3 (09-03-01, 5 n; 14-03-01, 1 c, 2 n, 6 a; 23-03-01, 4 c, 6 a; 30-03-01, 10 c), 5 (02-03-01, 2 n, 2 a; 09-03-01, 3 n, 1 a), 6 (23-02-01, 2 n; 14-03-01, 3 c; 23-03-01, $1 \mathrm{n}$ ). No había sido previamente registrada en el humedal, por lo que se constituye en un nuevo registro.

\section{TYRANNIDAE}

Tyrannus melancholicus (Vieillot, 1819); nv. Sirirí, Sirirí capa negra, Sirilí, paparote, Tirano tropical, Tropical kingbird.

Se observaron 6 individuos en los sectores 1 (09-03-01, 1 c; 14-03-01, 1 c, 1 v), 4 (09-03-01, 2 p), y 5 (09-03-01, 1 v). Previamente Valencia (1999) y Benítez-Castañeda (2000a) la habían citado para el humedal.

\section{FRINGILLIDAE}

Carduelis psaltria (Say, 1823); nv. Chisga capanegra, Jilguero menor, Lesser goldfinch.

Se observaron 2 individuos en el sector 4 (09-03-01, 2 p). No había sido previamente registrada para el humedal, por lo que se constituye en un nuevo registro.

\section{EMBERIZIDAE}

Sicalis flaveola (Linnaeus, 1756); nv. Canario basto, Pinzón azafranado, Saffron finch.

Se observaron 7 individuos, en los sectores 1 (09-03-01, 1 c, 2 p; 23-03-01 1 v) y 4 (23-02-01, 1 p, 2 v). Previamente Benítez-Castañeda (2000a) la había citado para el humedal.

Sicalis luteola (Sparrman, 1789); nv. Canario bogotano, Pinzón sabanero, Grasland yellowfinch.

Se observaron 20 individuos, en los sectores 1 (09-03-01, 2 c, 1 p; 23-03-01 1 v); 4 (23-02-01, 2 p, 2 v; 14-03-01 10 v) y 5 (02-03-01, 1 juv. p, 1 p; 30-03-01 2 v). Previamente BenítezCastañeda (2000a) la había citado para el humedal.

Zanotrichia capensis P. L. S. Muller, 1776; nv. Copetón, Gorrión andino, Rufous-collared sparrow.

Se observaron 155 individuos en los sectores 1 (02-03-01, 4 c, 3 v; 09-03-01, 2 c, 1 p; 14-0301, 5 v; 23-03-01, 2 c, 1 v, 1 p; 06-04-01, 2 c, 5 p; 10-04-01, 1 c); 2 (04-10-03, 2 p; 09-03-01, 2 p; 14-03-01, 1 p; 23-03-01, 1 c, 1 p; 06-04-01, 2 c, 1 p; 10-04-01, 1 v, 1 p); 3 (04-10-03, 2 p; 2303-01, 1 c; 06-04-01, 1 p); 4 (23-02-01, 6 p; 02-03-01, 2 p; 09-03-01, 1 v; 14-03-01, 1 v; 23-0301, 1 c, 4 p; 30-03-01, 2 v; 06-04-01, 1 c, 2 v, 1 p; 10-04-01, 2 v, 2 p); 5 (23-02-01, 3 p; 02-0301, 2 p; 09-03-01, 2 c, 2 v; 14-03-01, 3 c; 23-03-01, 3 c; 30-03-01, 3 c; 06-04-01, 2 c, 2 v; 1004-01, 4 c); y 6 (23-02-01, 11 c, 12 v; 02-03-01, 3 c; 09-03-01, 3 c; 14-03-01, 1 c, 1 p; 23-03-01, 1 c, 3 v, 2 p; 30-03-01, 1 c; 10-04-01, 2 v, 3 p). Previamente Valencia (1999) y BenítezCastañeda (2000a) la habían citado para el humedal.

\section{VIREONIDAE}

Vireo olivaceus (Linnaeus, 1766); nv. Vireo ojirrojo, Red-eyed vireo. 
Se observaron 11 individuos en el sector 6 (23-03-01, 5 v, 6 p). No había sido previamente registrada para el humedal, por lo que se constituye en un nuevo registro.

THRAUPIDAE

Piranga rubra (Linnaeus, 1758); nv. Cardenal abejero, Tangara veranera, Summer tanager.

Se observaron 4 individuos en el sector 1 (23-03-01, 2 v, 2 p). No había sido previamente registrada para el humedal, por lo que se constituye en un nuevo registro.

Piranga olivacea (Gmelin, 1789); nv. Cardenal escarlata, Tangara escarlata, Scarlet tanager.

Se observaron 5 individuos en el sector 1 (23-03-01, 3 v, 2 p). No había sido previamente registrada para el humedal, por lo que se constituye en un nuevo registro.

Rhamphocelus dimidiatus (Lafresnaye, 1837); nv. Cardenal Pico de Plata, Tangara dorsirroja, Crimson-backed tanager.

Se observaron 6 individuos en el sector 1 (23-03-01, 2 v, 4 p). No había sido previamente registrada para el humedal, por lo que se constituye en un nuevo registro.

\section{CATHARTIDAE}

Coragyps atratus (Berchstein, 1793); nv. Gallinazo negro, Chulo, Black vulture.

Se observó 1 individuo en el sector 2 (14-03-01, 1 v). Previamente Valencia (1999) la había citado para el humedal.

TOGLODYTIDAE

Cistothorus apolinari (Chapman, 1914); nv. Cucarachero de pantano, Soterrey de Apolinar, Chirriador, Apolinar's wren.

Se observaron 4 individuos en el sector 4 (02-23-01, 2 c, 2 p). Previamente Molina \& Osorio (1995) la habían citado para el humedal.

ANPHIBIA

HYLIDAE

Hyla labialis (Linnaeus, 1798); nv. Rana verde de la Sabana.

Se le observó en los sectores 1 (04-10-03, 2 ind. en percha) y 4 (02-23-01, 3 ind. en percha; 03-30-01, 2 ind. en percha; 04-06-01, 3 ind. en percha; 04-10-03, 2 ind. en percha). No registrada previamente, por lo que se constituye en un nuevo registro para el humedal.

PISCES

TRICHOMYCTERIDAE

Eremophilus mutissii (Humboldt, 1805); nv. Capitán de la Sabana.

Se le observó en los sectores 1 (04-10-03, 2 ind.) y 4 (02-23-01, 3 ind.). Previamente registrada para el humedal por Rodríguez-Mahecha et al. (2000) y Sáenz-Guarín (2001).

\section{CHARACIDAE}

Grundulus bogotensis (Humboldt, 1821); nv. Guapucha.

Se le observó en los sectores 2 (04-10-03, 2 ind.) y 5 (02-23-01, 2 ind.). Previamente registrada para el humedal por Rodríguez-Mahecha et al. (2000) y Sáenz-Guarín (2001).

\section{CYPRINODONTIDAE}

Carasius auratus (Linnaeus, 1758); nv. Bailarina, Pez velo, Rojito.

Se le observó en los sectores 1 (04-10-03, 2 ind.) y 4 (04-10-03, 2 ind.). No registrada previamente, por lo que se constituye en un nuevo registro para el humedal.

MAMALIA

CAVIDAE

Cavia anolaimae (J. A. Allen, 1916); nv. Curí. 
Se le observó en los sectores 5 (02-23-01, 2 ind.). No registrada previamente, por lo que se constituye en un nuevo registro para el humedal.

\section{MURIDAE}

Oeomys speciosus (J. A. Allen Chapman, 1893); nv. Ratón aterciopelado sabanero.

Se le observó en el sector 4 (02-23-01, 3 ind.; 03-30-01, 2 ind.). No registrada previamente, por lo que se constituye en un nuevo registro para el humedal.

Olygoryzomys fulvescens (de Saussure, 1860); nv. Ratón arrocero pigmeo occidental.

Se le observó en el sector 5 (04-06-01, 3 ind.; 04-10-03, 2 ind.). No registrada previamente, por lo que se constituye en un nuevo registro para el humedal.

\section{CONCLUSIONES}

\section{Fauna asociada al humedal y sus zonas de influencia:}

Los análisis de la información obtenida en los muestreos y en las observaciones del presente trabajo y los realizados por diferentes instituciones y personas entre 1999 y 2002 dentro del humedal, permiten hacer los siguientes comentarios y conclusiones:

Las observaciones estuvieron dedicadas principalmente a las especies que utilizan el humedal (97\% del total) y las áreas circunvecinas. Por tanto, el registro de especies cuya actividad está relacionada con los diferentes subsistemas, se realizó mientras se llevaba a cabo el traslado alrededor del humedal muestreado.

El total de familias ascendió a 22 (3 de peces, 1 de anfibios, 17 de aves y 2 de mamíferos) y el de especies a 34 (3 de peces, 1 de anfibios, 29 de aves y 3 de mamíferos) (Apéndice 1). De los registros, 6 son nuevos para el área de estudio (3 de peces, 1 de anfibios y 2 de mamíferos). Vale la pena resaltar que, con la única excepción de las aves, los demás taxa sólo han tenido muestreos esporádicos, por lo que el inventario ornitológico del área del Humedal de Santa María del Lago reviste una importancia especial para detectar las variaciones que se han presentado año a año. Los registros previos de aves ascendían a 28-30 especies, según Molina-Prieto y Osorio-Olarte (1995), Valencia (1999) y Benítez-Castañeda (2000a).

Las anotaciones de campo consignadas en la Apéndice 1 y en la lista anotada, permitieron confirmar el rango de distribución y ampliar los conocimientos que se tenían sobre la utilización de los diferentes hábitats. Previamente sólo se habían registrado las épocas de reproducción de una especie: el pato zambullidor pico rayado (Podilymbus podiceps) (Benítez-Castañeda, 2000b).

Las 13 especies que se registran por primera vez para el Humedal de Santa María del Lago y para sus áreas de influencia son: aves (Anas platyrhynchos, Anser anser, Diglossa humeralis, Piranga rubra, P. olivacea, Rhamphocelus dimidiatus, Stelgidopteryx ruficollis, Vireo olivaceus); anfibios (Hyla labialis); peces (Carasius auratus); mamíferos (Cavia anolaimae, Oeomys speciosus, Olygoryzomys fulvescens), los cuales se observaron especialmente en los sectores 4 y 5.

De las cuatro tinguas del Altiplano cundi-boyacense, en el Humedal de Santa María del Lago se registran tres especies: la amarilla (Fulica americana), la roja (Gallinula chloropus), y la azul (Porphino martinicus), las cuales se distribuyen en los seis sectores del humedal.

Aunque no se realizaron estudios detallados del efecto que causan las aves sobre el arbolado presente en el humedal, fue posible observar cómo las garzas reales (Egretta alba), las palomas (Columba livia, Zenaida auriculatus), las golondrinas (Hirudo rustica, Notocheilodon murina, Stelgidopteryx ruficolis) y las mirlas (Turdus furcater) lo ocupan en las diferentes horas del día, especialmente para hacer percha -la gran mayoría- o para vivir en las cortezas de los árboles presentes -específicamente en el eucalipto-.

En cuanto a la abundancia de las familias y las especies en las diferentes zonas, las que presentaron un mayor número fueron aquellas situadas en el sector 4 (19 familias, 28 especies) y en el sector 5 (12 familias, 19 especies) debido, al parecer, a la flora acuática y ribereña que está en un relativo buen estado.

En esta contribución se ha creído de utilidad agrupar las especies del área de estudio en endémicas (26), migratorias (8) e introducidas (4). 


\section{Parámetros biológicos, físicos y químicos de las aguas:}

Los 27 parámetros (8 físicos, 16 químicos, 3 biológicos) evaluados mediante análisis puntuales dentro del humedal, tanto por instituciones como por personas desde 1988 hasta el 2001, permiten hacer los siguientes comentarios y conclusiones:

Previo a los análisis, el humedal ha sufrido modificaciones, a veces severas, que han alterado en mayor o menor medida sus hábitats acuáticos y terrestres, lo cual impide una secuencia de la calidad de sus aguas en el tiempo.

Si bien los datos absolutos pueden servir para tener una idea de la calidad de las aguas del humedal, ni la cantidad de parámetros ni la metodología han sido uniformes o estandarizados, por tanto no hay una precisión comparable

El humedal carece de una batimetría detallada y confiable, al igual que carece de un estudio hidráulico que permita conocer cómo es la dinámica del sistema y, especialmente, cómo será su comportamiento después de que sean retiradas las aguas combinadas que actualmente son la fuente principal del recurso hídrico. El resto de agua proviene de seis puntos con tubería de aguas lluvias.

Para el humedal existen datos metereológicos complementarios medidos en el aeropuerto internacional El Dorado y en algunos humedales vecinos: temperatura ambiental media $14^{\circ} \mathrm{C}$, precipitación pluvial 840-915 mm, humedad relativa 78-86\%, evaporación 79.3-102.1 mm/mes y $1066.1 \mathrm{~mm} / \mathrm{año}$, vientos $2-4 \mathrm{~m} / \mathrm{s}$.

Es muy positivo que la caracterización se haya realizado en las etapas iniciales de un proyecto piloto de recuperación ambiental, precisamente cuando se están dando las bases de un manejo sustentable del humedal.

La caracterización realizada a comienzos del 2001, de tipo físico (3 parámetros), químico (15 parámetros) y biológico (1parámetro), permitirá ofrecer una amplia base de información que debe ser repetida periódicamente (cada 3 o 4 meses al año), con el fin de evaluar las mejoras que se deben ir dando a medida que se mejoran las condiciones del humedal.

Los contenidos de oxígeno indican los fenómenos de eutroficación que se llevan a cabo en el cuerpo de agua y sus orillas, lo cual, unido a especies indicadoras del perifiton hallado, permite clasificarlo actualmente como de tipo oligotrófico.

Los bajos niveles de DBO y DQO indican, por un lado, una reducida actividad del oxígeno y, por el otro, su consumo mínimo en las aguas del humedal.

Los fosfatos, nitritos, nitratos y sulfatos son, en general, bastante bajos y sus rangos en el humedal indican la concentración en diferentes puntos de la escasa materia orgánica al parecer por la acción antrópica, la dinámica del cuerpo de agua, la vegetación circundante, el régimen de vientos y la geomorfología actual.

Es muy alentador que las concentraciones de metales pesados como el cadmio, mercurio y plomo sean bajas, dada la peligrosidad de los compuestos metilados de dichos elementos tóxicos.

El comportamiento de las concentraciones de los coliformes tanto totales como fecales, clásicos indicadores de la calidad de las aguas especialmente para su uso, puede ser el reflejo de la hidrodinámica del humedal, el filtro creado por la vegetación flotante, las condiciones climáticas, pues este parámetro no se comporta como proveniente de las aguas servidas 0 negras que llegan al humedal.

Los muestreos siguientes permitirán evaluar poco a poco cómo el cuerpo de agua se comporta ante el proceso de recuperación de su área superficial (extracción de la vegetación flotante y retiro de las aguas combinadas que le llegan) y de la ronda del humedal.

Aún en los peores momentos del humedal, se destaca la presencia de fauna (aves, peces, anfibios, invertebrados, zooplancton) y flora asociada (macrófitas acuáticas y macrófitas emergentes, fitoplancton). Dichos organismos aprovechan directamente el agua y sus recursos. Tal presencia, por cierto alentadora, habla de la mejora constante que se debe y puede realizar en la calidad del agua del humedal.

\section{AGRADECIMIENTOS}


A Gonzalo Aristizábal y Carlos Colmenares (Fundación La Tingua) que acompañaron los recorridos de observación y aportaron valiosa información sobre las especies encontradas; a Jorge I. Hernández-Camacho (q.e.p.d.) de BIOCOLOMBIA, por las sugerencias al manuscrito original.

\section{LITERATURA CITADA}

ABO. 1999. Humedal Santa María del Lago. El Clarinero (Asoc. Bogotana de Ornitología), 20: 3-4.

Abril, P. M. Arcos, A. Baigari, S. Bogotá, J. Cadena, Z. Daza, N. Dimaté, J. Fernández, P. Quintero, N. Ruíz M. Santos. 2000. Factores estructurales y funcionales del Humedal de Santa María del Lago, octubre 2000 (Estación 1). Fac. Biología Marina. Univ. Jorge Tadeo Lozano. Santa Fe de Bogotá D. C. Inf. Técnico Asignatura de Ecología (Profesor Gabriel Pinilla, Asesora Marcela Cañón).

Álvarez-López, H. 1999. Guía de aves de la Reserva Natural Laguna de Sonso. CVC. Imágenes de la Naturaleza. Cali (Valle) Colombia, 107 p.

Arias, A, P. Bueno, J. P. Camargo, J. D. Castañeda, G. X. Díaz, M. A. Duarte, M. Forero, A. Gartner, L. Gómez, A. Lugo, P. Manrique, M. del A. Martínez, A. Matíz, A. M. Millán, J. C. Millán, E. Ortiz, C. J. Polo, M. A. Ramírez, A. Recalde, F. Reyes, M. C. Rosso, A. Sandoval, A. M. Suárez, C. Torres, D. A. Varilla \& C. Vásquez. 2001. Análisis ecológico Humedal de Santa María del Lago, abril 2001. Fac. Biología Marina. Univ. Jorge Tadeo Lozano. Santa Fe de Bogotá D. C. Inf. Técnico Asignatura de Ecología (Profesor Gabriel Pinilla, Asesora Marcela Cañón), s. p.

Barrera, A., M. Díaz, J. García, G. Guarín, G. Guerrero, K. Gutiérrez, L. Mantilla, A. Rodríguez, D. Vélez \& S. Sánchez. 2000. Diagnóstico ambiental del Humedal de Santa María del Lago, octubre 2000 (Estación 2). Fac. Biología Marina. Univ. Jorge Tadeo Lozano. Santa Fe de Bogotá D. C. Inf. Técnico Asignatura de Ecología (Profesor Gabriel Pinilla, Asesora Marcela Cañón), 48 p.

Benítez-Castañeda, H. D. 2000a. Registro parcial de aves en el humedal Santa María del Lago: $2-6$ de octubre. Lic. de Biología Univ. Distrital Francisco José de Caldas. Santa Fe de Bogotá D. C. Inf. Técnico, $4 \mathrm{p}$.

Benítez-Castañeda, H. D. 2000b. Descripción, costumbres y comportamiento del zambullidor pico rayado Podilymbus podiceps. Lic. de Biología Univ. Distrital Francisco José de Caldas. Santa Fe de Bogotá D. C. Inf. Técnico, s. p.

Benítez-Castañeda, H. D. 2001. Observaciones del comportamiento reproductivo y alimentario del zambullidor pico rayado Podilymbus podiceps (Aves: Podicipedidae) en los humedales Santa María del Lago y La Florida. Facultad de Ciencias de la Educación. Univ. Distrital Francisco José de Caldas, s.p.

Bohórquez, C. I., C. D. Cadena, S. De La Zerda, M. Hernández, M. Kelsey, D. Knapp, L. Rosselli, F. G. Styles \& I. D. Valencia. 2000. Aves de la Sabana de Bogotá - Guía de Campo-. Asoc. Bogotana de Ornitología / CAR / Audubon América Latina. Santa Fe de Bogotá D. C. (Colombia), 276 p.

Calvachi-Zambrano, B. 2003. La fauna de los humedales. De la majestuosidad de los mastodontes al oportunismo de las ratas, Vol. 1 pp. 109-138 In: Guarnizo, A. \& B. CalvachiZambrano (coords.) Los Humedales de Bogotá y la Sabana. Empresa de Acueducto y de Alcantarillado de Bogotá / Conservación Internacional-Colombia. Bogotá D. C. (Colombia), 264 p.

CAR. 1996. Resultados de análisis fisicoquímicos. Propuesta Plan de Manejo Ambiental del Humedal Santa María del Lago, mayo 21. Santa Fe de Bogotá D. C. Inf. Técnico, s. p.

Cely, J., H. D. Benítez-Castañeda, L. J. Vargas, C. Villalobos, A. J. Niño, \& J. García. 2002. Santa María del Lago, In: II Contero de Aves en los Humedales de la Sabana de Bogotá y el I Censo Nacional de Aves, ABO / Inst. Alexander von Humboldt / CALIDRIS. El Clarinero, 31 (mayo). 
EAAB. 1988. Resultados de análisis fisicoquímicos. Propuesta Plan de Manejo Ambiental del Humedal de Santa María del Lago, abril. Empresa de Acueducto y Alcantarillado de Bogotá. Bogotá D. E. Inf. Técnico, s. p.

HIDROANÁLISIS-PRODICON LTDA. 2001. Caracterización fisicoquímica e hidrobiológica. Muestreo puntual del Humedal Santa María del Lago, febrero 22. Bogotá D. C. Inf. Técnico, s. p.

Hilty, S. L. \& W. L. Brown. 1986. Birds of Colombia. Princenton University Press. Princenton (N. J.) USA, $836 \mathrm{p}$.

Meyer de Schauensee, R. 1949. The birds of the Republic of Colombia, Part 2. Caldasia, 5 (23): 381-644.

Molina-Prieto, L. F. \& J. Osorio-Olarte. 1995. Guía de aves de Santa Fe de Bogotá. DAMA IAMSFB. Gráficas de La Sabana Ltda. Santa Fe de Bogotá D. C. (Colombia), 225 p.

Osorio-Olarte, J., E. Uribe-Botero \& L. F. Molina-Prieto. 1997. Cerros, humedales y áreas rurales de Santa Fe de Bogotá. DAMA /AMSFB. Santa Fe de Bogotá D. C. (Colombia), 190 p.

Olivares, A. 1959. Aves migratorias en Colombia. Rev. Acad. Colomb. Cienc., 10 (41): 371-442. 1969. Aves de Cundinamarca. Dir. Divul. Cultural, Univ. Nacional de Colombia. Bogotá D. E. (Colombia), 425 p. 1973. Las Ciconiformes colombianas (garzas, coclearios, ibis, cigüeñas, espátulas, flamencos). Edic. Tercer Mundo / PROYSER. Bogotá D. E. (Colombia), 303 p. 1982. Aves de la Orinoquia. 2a Ed. UNC-ICN / CDI Las Gaviotas. Impr. Nacional. Bogotá D. E. (Colombia), 127 p.

Pantaleón, A., S. Quiroga, D. Rodríguez \& J. Tello. 1999. Evaluación preliminar del estado actual del ecosistema Humedal de Santa María del Lago, a través de indicadores acuáticos durante los meses de septiembre a noviembre de 1999. Fac. Biología Marina. Univ. Jorge Tadeo Lozano. Santa Fe de Bogotá D. C. Inf. Técnico Asignatura de Ecología (Profesor Gabriel Pinilla), $18 \mathrm{p}$.

Phelps Jr., W. H. \& R. Meyer de Schauensee. 1979. Una guía de las aves de Venezuela. Gráficas Armitano C.A. Caracas, (Venezuela), 484 p.

Pinto, M., H. Zúñiga \& O. M. Torres. 2001. Estudio sistemático del género Cavia Pallas, 1766 (Rodentia: Caviidae) en Colombia. Revisión del registro arqueológico colombiano. Acad. Colomb. Cienc. Exactas, Físicas y Naturales. Colec. Jorge Alvarez Lleras, 21: 1-212.

Rengifo, L. M., A. M. Franco-Maya, J. D. Amaya-Espinel, G. H. Catan \& B. López-Lanús (eds.). 2000. El libro rojo de las aves de Colombia. La Serie de Libros Rojos de Especies Amenazadas de Colombia. IIBAVH / MINAMBIENTE / Cl-Colombia / ICN-UNC / Santa Fe de Bogotá D. C. (Colombia), 562 p.

Rodríguez-Mahecha, J. V. 1982. Aves del Parque Nacional Natural los Katíos: ChocóColombia. Proyecto ICA / INDERENA / USDA. Talleres del INDERENA. Bogotá D. E. (Colombia), 328 p.

Rodríguez-Mahecha, J. V., J. I. Hernández-Camacho T. R. Defler, A. Alberico, R. B. Mast, R. Mittermeier \& A. Cadena. 1995. Mamíferos colombianos: sus nombres comunes e indígenas. Conservación Internacional. Occasional Paper In Conservation Biology, 3: 1-56.

Rodríguez-Mahecha, J. V., R. H. Orozco-Rey, V. H. Vásquez-Vásquez, B. Calvachi-Zambrano, J. Mendoza-Sabogal, R. Alvarez-León, G. Camacho-Camacho, J. I. Hernández-Camacho \& U. Smith-Mumm. 2000. Síntesis del estado actual de los humedales de la Sabana de Bogotá. Cap. 5 Santa María del Lago (pp. 64-72). Proyecto Recuperación de los Humedales de la Sabana de Bogotá: Alternativa hacia su Viabilidad Ecológica y Social. Empresa de Acueducto y de Alcantarillado de Bogotá / Conservación Internacional-Colombia. Santa Fe de Bogotá D. C. (Colombia), 192 p.

Sáenz-Guarín, J. E. 2001. Diagnóstico bioecológico del estado actual de la ictiofauna en el humedal Santa María del Lago, Bogotá D. C., p. 59 In: Mem. Resúmenes VI Simp. Colombia de Ictiol. ACICTIOS / UNC-DB / ACCEFN. Bogota D. C. (Colombia), agosto 16-18, 60 p. 
Salaman, P., T. Cuadros, J. G. Jaramillo \& W. H Weber. 2001. Lista de chequeo de las aves de Colombia. Sociedad Antioqueña de Ornitología. Medellín (Ant.) Colombia, 116 p.

Schmidt-Mumm, U. 1998. Vegetación acuática palustre de la Sabana de Bogotá y plano del río Ubaté. Ecología y taxonomía de la flora acuática y semi-acuática. Tesis de Magíster. Depto. de Biología, Facultad de Ciencias. Univ. Nacional de Colombia.

Valencia, I. D. 1999. Registro de aves del humedal Santa María del Lago: 26 de septiembre. Asoc. Bogotana de Ornitología. Santa Fe de Bogotá D. C. Inf. Técnico, 2 p.

Wyatt, C. W. 1871. Notes on some of the birds of the United States of Colombia. Ibis, April: 113131; July: 319-335; October: 373-384.

Zerda-Ordoñez, E. 1992. Guía de las aves en el Jardín Botánico "José Celestino Mutis". Col. Francisco José de Caldas, Vol. 1. Santa Fe de Bogotá D. C. (Colombia), 154 p.

Apéndice 1. Especies encontradas en el Humedal de Santa María del Lago, entre febrero 23 y mayo 31 de 2003. E= Endémico, I=Introducido, M=Migratorio; Fuentes previas: (1= MolinaPrieto y Osorio-Olarte, 1995; 2=Valencia, 1999; 3= Benítez-Castañeda, 2000a; 4= RodríguezMahecha et al., 2001; 5= Sáenz-Guarín, 2001; 6= Presente estudio)

\begin{tabular}{|c|c|c|c|}
\hline Nombre Científico & Nombre Vernacular & E-I-M & Fuentes \\
\hline \multicolumn{4}{|l|}{ Aves } \\
\hline Anas platyrhynchos & Pato doméstico & $\mathrm{I}$ & 6 \\
\hline Agelaius icterocephalus & Monjita bogotana & $E$ & $1,2,3$ \\
\hline Bubulcus ibis & Garza del ganado & $\mathrm{M}$ & 2,3 \\
\hline Butorides striatus & Garza castaña & $\mathrm{M}$ & 2,3 \\
\hline Carduelis psaltria & Chisga capa negra & $E$ & 6 \\
\hline Cistothorus apolinari & Cucarachero de pantano & $E$ & 1 \\
\hline Colibri coruscans & Colibrí orejiverde & $E$ & 1,3 \\
\hline Columba livia & Paloma común, Paloma de Castilla & I & 3 \\
\hline Coragyps atratus & Gallinazo negro & $\mathrm{M}$ & 2 \\
\hline Anser anser & Ganso común, Ganso europeo & $\mathrm{I}$ & 6 \\
\hline Diglossa humeralis & Carbonero común & $E$ & 6 \\
\hline Egretta alba & Garza real & $\mathrm{M}$ & 2,3 \\
\hline Forpus conspicillatus & Loro de anteojos & $E$ & 2 \\
\hline Fulica americana & Tingua pico amarillo & $E$ & 2,3 \\
\hline Gallinula chloropus & Tingua pico rojo & $E$ & $1,2,3$ \\
\hline Hirudo rustica & Golondrina negra & $\mathrm{M}$ & 2,3 \\
\hline Molothrus bonariensis & Maicero & $E$ & 2,3 \\
\hline Notocheliodon murina & Golondrina vientrigrís & $\mathrm{M}$ & 2,3 \\
\hline Piranga rubra & Cardenal abejero & $E$ & 6 \\
\hline Piranga olivacea & Cardenal escarlata & $E$ & 6 \\
\hline Podilymbus podiceps & Zambullidor pico rayado & $E$ & 2,3 \\
\hline Porphirio martinica & Tingua pico azul & $E$ & $1,2,3$ \\
\hline Rhamphocelus dimidiatus & Cardenal pico de plata & $E$ & 6 \\
\hline Sicalis flaveola & Canario & $E$ & 3 \\
\hline Sicalis luteola & Canario bogotano & $E$ & 3 \\
\hline Stelgidopteryx ruficollis & Golondrina pecho marrón & $\mathrm{M}$ & 6 \\
\hline Turdus fuscater & Mirla & $E$ & 3 \\
\hline Tyrannus melancholicus & Sirirí & $E$ & 2,3 \\
\hline Vireo olivaceus & Vireo ojirrojo & $\mathrm{M}$ & 6 \\
\hline Zanotrichia capensis & Copetón & $\mathrm{E}$ & 2,3 \\
\hline Zenaida auriculata & Torcaza bogotana & $E$ & 2,3 \\
\hline \multicolumn{4}{|l|}{ Pisces } \\
\hline Carasius auratus & Bailarina, Rojito, Goldfish & $\mathrm{I}$ & 6 \\
\hline Eremophilus mutissii & Capitán de la Sabana & $E$ & 4,5 \\
\hline Grundulus bogotensis & Guapucha & $E$ & 4,5 \\
\hline \multicolumn{4}{|l|}{ Anphibia } \\
\hline Hyla labialis & Ranita verde & $E$ & 6 \\
\hline \multicolumn{4}{|l|}{ Mammalia } \\
\hline Cavia anolaimae & Curí & $E$ & 6 \\
\hline Oeomys speciosus & Ratón aterciopelado sabanero & $E$ & 6 \\
\hline Olygoryzomys fulvescens & Ratón arrocero pigmeo occidental & $E$ & 6 \\
\hline
\end{tabular}


Apéndice 2. Comparación de la información hidrológica (biológica, física y química) disponible sobre el Humedal de Santa María del Lago (1988-2001). (1) EAAB (1988); (2) CAR (1996); (3) Pantaleón et al. (1999); (4) Abril et al. (2000); (5) Barrera et al. (2000); (6) Arias et al. (20001); (7) Hidroanálisis - Prodicon Ltda. (2001)

\begin{tabular}{|c|c|c|c|c|c|c|c|}
\hline $\begin{array}{l}\text { PARÁMETRO } \\
\text { (unidades) }\end{array}$ & 1 & 2 & 3 & 4 & 5 & 6 & 7 \\
\hline $\begin{array}{l}\text { Temperatura ambiente } \\
\qquad\left({ }^{\circ} \mathrm{C}\right)\end{array}$ & - & - & - & - & - & - & 15 \\
\hline $\begin{array}{c}\text { Temperatura agua } \\
\left({ }^{\circ} \mathrm{C}\right)\end{array}$ & - & $15.0-20.5$ & $16-20$ & $13-17$ & $11-14.5$ & $17.0-17.3$ & $11-19$ \\
\hline $\mathrm{pH}$ unid. & $7.09-7.57$ & 6.8 & $5.5-8.2$ & 6.5 & $6.2-6 . .5$ & $6.02-6.20$ & $6.87-7.15$ \\
\hline $\begin{array}{c}\text { Conductividad } \\
\text { (microsimens / cm) }\end{array}$ & - & - & $210-400$ & - & - & $218-241$ & $200-280$ \\
\hline $\begin{array}{c}\text { Oxígeno disuelto } \\
\mathrm{mg} / \mathrm{l}\end{array}$ & - & $6.68-13.44$ & $3.0-14.2$ & $0.7-0.9$ & $0.49-1.5$ & $0.55-1.0$ & $0.0-1 . .2$ \\
\hline Color & - & - & - & - & - & - & $\begin{array}{l}\text { Amarillento- } \\
\text { semiturbio }\end{array}$ \\
\hline Grasas (mg/l) & - & $10.6-24.38$ & - & - & - & - & - \\
\hline Sobrenadante & - & - & - & - & - & - & No-Iridiscente \\
\hline Transparencia (cm) & - & - & - & $40-60$ & - & $0.65-1.25$ & - \\
\hline $\begin{array}{l}\text { Velocidad de la } \\
\text { Corriente (m/s) }\end{array}$ & - & - & - & $0.04-2.46$ & - & - & - \\
\hline $\begin{array}{c}\text { Acidez } \\
(\mathrm{mg} / \mathrm{l} \text { como } \mathrm{CaCO} 3)\end{array}$ & - & & - & - & - & - & $16-36$ \\
\hline
\end{tabular}




\begin{tabular}{|c|c|c|c|c|c|c|c|}
\hline $\begin{array}{c}\text { Alcalinidad } \\
(\mathrm{mg} / \mathrm{l} \text { como } \mathrm{CaCO} 3)\end{array}$ & - & - & - & - & - & - & $82-108$ \\
\hline DBO-5 (mg/l O2) & $9-18$ & - & - & - & 9 & - & $12-28$ \\
\hline DQO (mg/l O2) & $28-368$ & $38-104.9$ & - & - & - & - & $30-90$ \\
\hline $\begin{array}{c}\text { Fosfatos } \\
(\mathrm{mg} / \mathrm{l} \text { como } \mathrm{P})\end{array}$ & - & $0.033-0.258$ & $0.5-2.5$ & 0.25 & 9.5 & $1.39-1.76$ & $0.08-2.19$ \\
\hline $\begin{array}{c}\text { Nitratos } \\
(\mathrm{mg} / \mathrm{l} \text { como } \mathrm{N})\end{array}$ & - & $0.281-1.32$ & $1.0-5 . .0$ & - & 10.0 & $0.29-9.46$ & $<0.01-0.04$ \\
\hline $\begin{array}{c}\text { Nitritos } \\
(\mathrm{mg} / \mathrm{l} \text { como } \mathrm{N})\end{array}$ & - & $0.003-0.017$ & $0.01-0.05$ & - & 0.15 & $0.022-0.0759$ & $<0.001-0.018$ \\
\hline $\begin{array}{c}\text { Amoníaco } \\
(\mathrm{mg} / \mathrm{l} \text { como } \mathrm{N})\end{array}$ & $1-5$ & $0.22-2.5$ & - & 0.4 & 0.2 & - & - \\
\hline Cadmio (mg/l Cd) & - & - & - & - & - & - & $<0.001$ \\
\hline Mercurio (ug/l Hg) & - & - & - & - & - & - & $<0.001$ \\
\hline Plomo (ug/l Pb) & - & - & - & - & - & - & $<0.01$ \\
\hline $\begin{array}{l}\text { Sólidos disueltos } \\
\text { (mg/l) }\end{array}$ & - & $0.06-0.226$ & - & - & - & - & $157-257$ \\
\hline $\begin{array}{l}\text { Sólidos sedimentables } \\
\text { (mg/l) }\end{array}$ & $32-192$ & $0.12-0.321$ & - & - & - & - & $<0.1-0.1$ \\
\hline Sulfatos (mg/l SO4) & - & - & $0.0-25.0$ & - & - & - & $<0.1-295$ \\
\hline Coliformes fecales & - & - & - & - & - & - & $240-15.000$ \\
\hline
\end{tabular}




\begin{tabular}{|c|c|c|c|c|c|c|c|}
\hline (UFC/100ml) & & & & & & & \\
\hline $\begin{array}{l}\text { Coliformes totales } \\
\text { (UFC/100 ml) }\end{array}$ & - & $30.000-36.000$ & - & - & - & - & $23.000-75.000$ \\
\hline Diagnóstico & - & - & Eutrófico & - & Eutrófico & Oligotrófico & Oligotrófico \\
\hline
\end{tabular}

